\title{
Design of Tracing Intelligent Vehicle Based on Image Processing
}

\author{
Chongyang Lv, Yuxin Wang
}

North China Electric Power University, Baoding 071000, China

Keywords: tracking, CCD camera, mage acquisition

\begin{abstract}
In order to realize the function of intelligent vehicle tracking according to different path types, a tracking vehicle based on automobile model is developed. In the design of the intelligent vehicle, the image processing can directly affect the performance of the intelligent vehicle. This paper describes the design of the intelligent vehicle system, and introduces the design of the racing hardware system, including the power module, the image acquisition module, the motor drive Module, speed acquisition module, etc., and the black line recognition algorithm based on CCD camera is put forward and the corresponding control strategy is introduced in detail. In addition, the paper also elaborates the adjustment method of the mechanical structure of the car, and carries on a lot of hardware and software tests. The experimental results show that the system design scheme is feasible.
\end{abstract}

\section{System hardware design}

System hardware design can be said that the entire smart car design basis and the most important. The correct hardware design direction and ideas, the system is stable and reliable basis, powerful hardware system, more software system to provide a strong protection. Hardware is the foundation, so the design is very easy to affect the stability of the car model and flexible steering performance.

\section{System software design}

Hardware systems and machinery are in the bottom, is the basis of the entire system, and the software system is based on mechanical performance. This module is essential for the entire system, efficient and stable software program is the basis of stable and rapid hatchback. And image acquisition and processing is the core of this module, the following is the basic process of software design module.

\section{Adjustment and optimization of mechanical structure}

Body structure is a very important aspect of the hardware, from the control point of view, this part is both the system's actuator and the controlled object. Optimization of the chassis parameters and optimization of the front wheel parameters can ensure that the body in the mechanical structure has a very good performance, it has a strong ability to perform, its importance no less than a good control strategy to ensure that the object of light And flexible, the same is conducive to improving the control effect.

In order to make smart cars in the straight and corners can be high-speed and stable driving, and turn comparison smart, fast, in addition to the corresponding software and hardware circuit design, the model of the mechanical structure of the car .It also has an important impact. Therefore, it should be based on the basic principles of vehicle construction, through repeated tests, indeed .Set the smart car models the main pin angle, the front beam, the main pin dip angle of the best parameters, increase the smart car stability qualitative, so that it can be in the role of intelligent control system under the fast and stable driving. 


\section{System implementation and experiment}

The direction of the car control, is the use of the camera to extract the black line location information, and then through a set the control strategy, so that the car can track the black line in real time, automatic control steering wheel driven front wheel steering complete hunt. The car is a variable system in the process of driving one with intelligent of the car must be able to adjust the parameters according to the characteristics of the track to the best to achieve optimal control. To think to achieve the direction of the car adaptive control, we must first identify the path that is to be able to determine Track the shape of the characteristics of the track to calculate the radius of curvature. And then try to control the curvature of the track radius and control system of the car to turn the PWM signal between the establishments of the corresponding mathematical relationship. As a result, in the car into the bend it is possible to quickly calculate the desired PWM signal to determine the steering of the steering gear by determining the size of the curve. This is the basic idea of our direction control.

Racing car in the process of turning, the camera and track space position as shown in Figure 1.

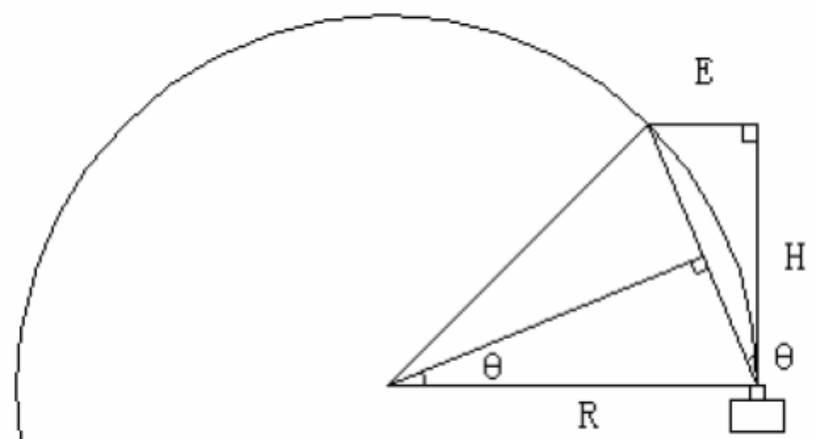

Figure 1. The camera and track space position

The derivation of the radius of curvature $\mathrm{R}$ is as follows:

By the trigonometric function:

$$
\frac{E}{\sqrt{E^{2}+H^{2}}}=\frac{\sqrt{E^{2}+H^{2}}}{2 R}
$$

Then we could get that:

$$
R=\frac{E^{2}+H^{2}}{2 E}
$$

Thus we get the formula for turning the radius. After the camera mounting position is fixed, it is converted in the world coordinate system, can calculate the distance of $0.35 \mathrm{~m}$. Thus, we get the formula for turning the radius. In the path recognition, we only take the camera collected line 7 data for direction control. In the above formula, E for the camera to collect the black line with the location racing center line between the horizontal offset; $\mathrm{H}$ for the camera to collect images in the first 7 lines of data from the front of the car distance, after the camera mount position is fixed, it will be converted in the world coordinate system, can calculate the distance is about $0.35 \mathrm{~m}$; $\mathrm{R}$ is the radius of curvature of the curve. In this relation, $\mathrm{H}$ is a known quantity, bending the magnitude of the curvature radius $\mathrm{R}$ depends only on the horizontal deviation of the black line position and the racing centerline (Figure 2). 


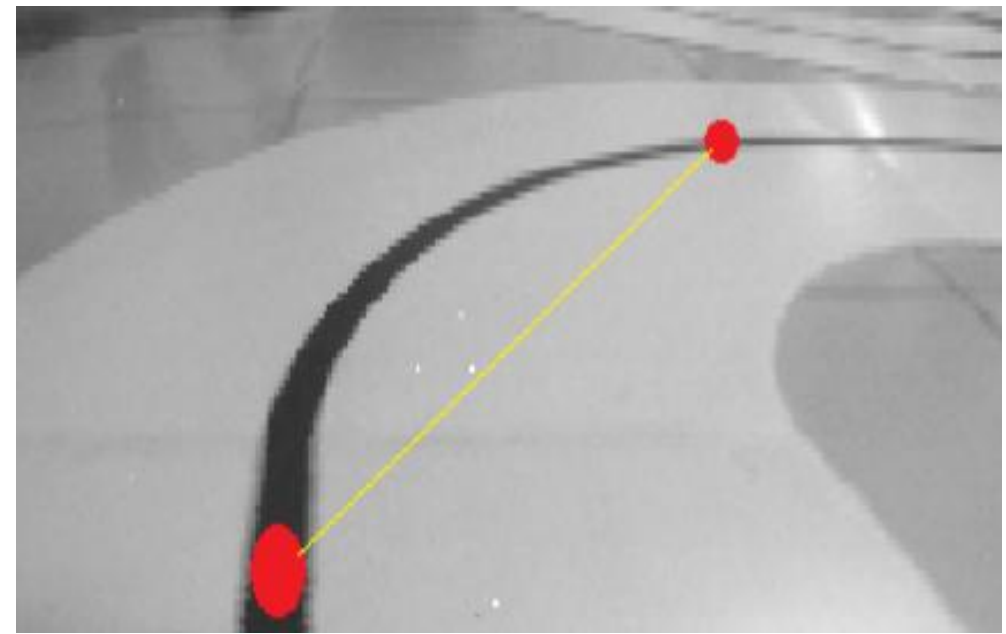

Figure 2. The black line with the location racing center line

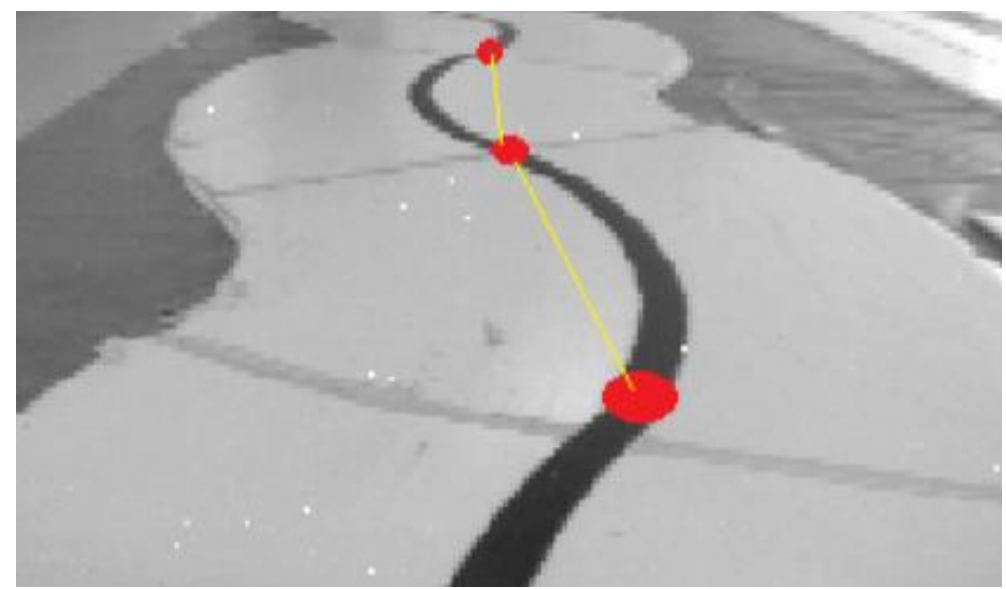

Figure 3. The horizontal deviation of the black line position and the racing centerline.

In order to be able to better optimize the realization of the corners of the cut and small $\mathrm{S}$ bent straight, we turned to the PID Correction, in the previous PID_out basis coupled with the slop control PID_outx, and to PID_out.

From the originImage [i] [j] start Right to judge each point(Image [i] [j +3$]$ - last point $)>$ threshold Assign $\mathrm{i}+\mathrm{k} / 2$ to the left edge of the black line The origin moves right by a point from Image [i] $[\mathrm{j}+$ 1] starts judgment The origin moves right by a point from Image [i] [j +1$]$ starts judgment Scan the origin 20 .Technical Report on the Sixth National College Students' Intelligent Automobile Competition On the rudder reference line on the 2 coefficient, so that the realization of Figure 3 shown in the cornering path.

PID_outx $=($ g_BlackPositionCenter [i] -g_BlackPositionCenter $[\mathrm{i}+\mathrm{ZX}]) * \mathrm{FD}$;

Servo_out $=$ PID_out $*(1+(\mathrm{i}-\mathrm{KK}) *(\mathrm{i}-\mathrm{KK}) / 20)+$. PID_outx ;

\section{Summary and outlook}

It has been tested and researched for the racing image acquisition and black line extraction algorithm, which laid the foundation for the fast and accurate tracking of the black line of the car system. At the same time, it improved the code in the experiment, adjusted the body structure, Road slowdown, to ensure that the shortest time to finish the whole process. 


\section{References}

[1] CHEN Meng-yuan, SUN Shu-cheng, WANG Hu. The Design and Research of Tracing Smart Car Based on Image Recognition. Journal of Chongqing Institute of Technology, 2013, 27(3), 80-84+103.

[2] LI Xu-dong, LIAO Zhong-hao, MENG Jiao. Intelligent Vehicle Control System Design and Realization Based on CMOS Camera. Journal of Jilin University (Information Science Edition), 2013,(04):414-418.

[3] XIAO Wen-jian LI Yong-ke. Design of intelligent vehicle based on incremental PID control algorithm. Information Technology, 2012, (10):125-127. 